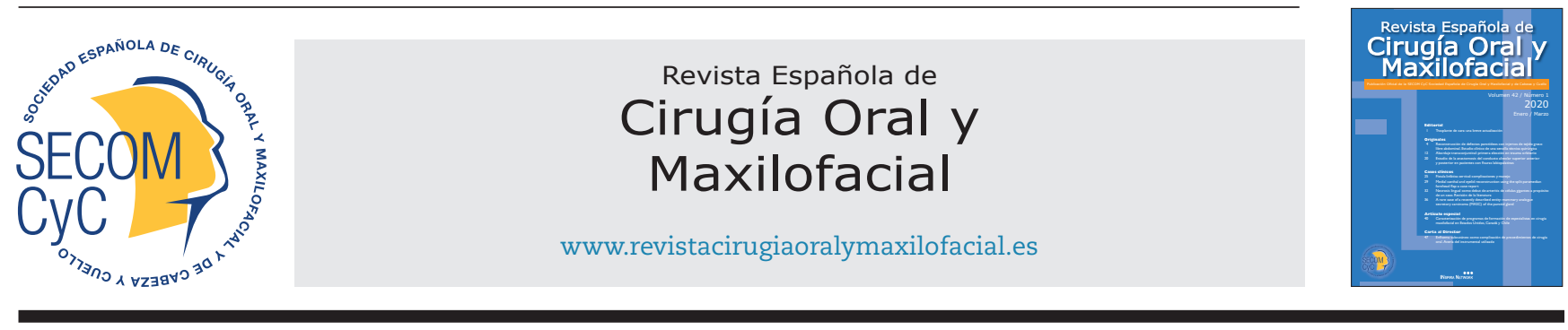

\title{
Editorial
}

\section{Trasplante de cara: una breve actualización}

Desde la realización del primer trasplante facial (TF) en Francia por Devauchelle y cols. en $2005^{1}$, se han referido hasta ahora más de una cuarentena de casos que, en su conjunto, han arrojado unos esperanzadores resultados inmunológicos, psicológicos, funcionales y estéticos, los cuales han puesto de manifiesto la viabilidad de este exigente y excepcional procedimiento ${ }^{2,3}$. Recientemente se ha conmemorado el décimo aniversario del primer trasplante de cara realizado en Andalucía, segundo en nuestro país y decimoprimero en el mundo, el cual tuvo lugar en enero de 2010 y en el que tuve el privilegio de participar junto a un nutrido grupo de cirujanos orales y maxilofaciales y cirujanos plásticos en el Hospital Universitario Virgen del Rocío de Sevilla ${ }^{4}$. Con el recuerdo de aquel hito, este editorial pretende realizar una breve actualización, partiendo de los resultados publicados por los equipos pioneros en TF, así como exponer algunas consideraciones sobre los principales resultados conseguidos y los próximos retos pendientes que plantea este nuevo y fascinante campo en constante evolución.

La comunidad científica involucrada en la cirugía reconstructiva de cabeza y cuello ha mantenido, desde sus comienzos, un enorme interés en la génesis, desarrollo y evolución de esta técnica. Hasta la fecha, ninguna otra técnica de cirugía reconstructiva había demostrado la capacidad de corregir y reparar tan variados y complejos defectos y deformidades anatómicas y funcionales de la cara. Sin embargo, a pesar de su constatado éxito, el TF ha generado, al mismo tiempo, un extenso debate científico. En esencia, el procedimiento técnico de un TF es similar al de cualquier otro complejo procedimiento microquirúrgico reconstructivo al alcance de numerosos hospitales, al cual hay que sumar conocimientos en planificación y fijación ósea maxilofacial, cirugía estética facial, de parótida y base de cráneo, incluyendo la disección de nervios faciales ${ }^{3}$. La estructura de la mayoría de los aloinjertos ha comprendido los dos tercios inferiores de la cara, especialmente la zona central perioral y periocular, conteniendo en algunos casos la frente, párpados y cuero cabelludo, así como el hueso maxilar, la mandíbula y los dientes ${ }^{5}$. El quid aquí radica en la exacta programación y ejecución quirúrgica, teniendo siempre en cuenta que hay que asegurar la adecuada perfusión y el aporte vascular del aloinjerto, el conocimiento de los angiosomas y la vascularización de los tejidos de la cara.

La mayoría de las indicaciones para un TF se han focalizado en pacientes que fueron víctimas de algún tipo de severo trauma o secuela grave que produjera un daño tisular extenso en el territorio centro-facial y en los que previamente fracasaron otros procedimientos de reconstrucción convencionales ${ }^{6}$. El objetivo se basa en restablecer las funciones para hablar, tragar y mover la musculatura mímica, así como en ofrecer mejoras estéticas que permitan a esos pacientes llevar una vida social normal. Aunque las características singulares de cada uno de los pacientes intervenidos de un TF impiden realizar un análisis estructurado, los resultados globales han sido convincentes ${ }^{7}$.

Los principales puntos de controversia se derivan de la terapia inmunosupresora y toxicidad farmacológica que pueden conducir a trastornos metabólicos, infecciones oportunistas y mayor incidencia de tumores malignos, el posible rechazo crónico y los costes económicos asociados 8,9 . De los 44 casos de TF conocidos en la actualidad, al menos 6 pacientes han fallecido como resultado de un rechazo inmunológico crónico, insuficiencia sistémica multiorgánica, infección, cáncer o suicidio ${ }^{10-12}$.

Una cuestión clave ampliamente debatida ha sido la obligatoriedad de someter a estos pacientes a un tratamiento inmunosupresor para toda la vida, con el consiguiente aumento del riesgo de desarrollar complicaciones ${ }^{13}$, teniendo en cuenta que esta "no es una cirugía para salvar vidas", a diferencia del trasplante de órganos sólidos que suele tener una indicación urgente para "realmente salvar la vida" de una persona. Desde tal punto de vista ético, los riesgos de exponer a los receptores a la terapia inmunosupresora podrían ser mayores que los beneficios del procedimiento. Sin embargo, todos los equipos de trasplantes han 
comunicado que el restablecimiento de las capacidades funcionales y la restauración de la cara "han cambiado la vida de los pacientes", y que se ha producido una mejora considerable en su calidad de vida ${ }^{14}$.

Los candidatos a TF se encuentran entre aquellos pacientes que presentan un daño tisular extenso en la cara y en los que con anterioridad han fracasado los procedimientos de reconstrucción habituales ${ }^{6}$. Desafortunadamente, los numerosos pacientes con cáncer avanzado de cabeza y cuello que se someten a un extenso procedimiento ablativo para tratar su enfermedad oncológica son malos candidatos para un TF, dado que seguramente tendrán un elevado riesgo de recidivar o desarrollar otro cáncer a consecuencia de la inmunosupresión. Un problema ético, todavía no resuelto, se plantearía si se produjera la pérdida total del aloinjerto como resultado de una complicación quirúrgica o un rechazo irreversible ${ }^{2}$. Ante semejante escenario adverso, quedarían muy pocas opciones reconstructivas para ese paciente ya que, hipotéticamente, se llegaría a una situación de salida mucho peor que la de partida ${ }^{15}$.

Es esencial una evaluación psicológica exhaustiva del receptor antes de incluirlo en un programa de $\mathrm{TF}^{16,17}$. Una contraindicación clara son los trastornos psicológicos que menoscaben la capacidad del receptor para afrontar el protocolo inmunosupresor o de distinguir una expectativa realista de lo que la cirugía puede ofrecer. El consentimiento informado previo al TF requiere una clara comprensión de los riesgos de la cirugía, de los aspectos psicológicos de recibir "la cara de otro individuo", de la terapia inmunosupresora y del posible rechazo crónico del aloinjerto. Los pacientes con un historial psiquiátrico dilatado o quienes han sufrido una lesión autoinfligida añaden otras consideraciones de especial complejidad, que son objeto de un debate aún no solventado.

Al mirar hacia atrás con la perspectiva de la década que ha transcurrido desde la realización de nuestro TF, cabe constatar con profunda satisfacción los enormes progresos conseguidos mundialmente en este novedoso campo. Pero a la vez conviene reconocer que todavía persisten numerosos problemas y desafíos y que quedan aspectos decisivos no resueltos para poder precisar el verdadero papel que el TF va a desempeñar en el futuro de la cirugía reconstructiva de cabeza y cuello. Pese al escepticismo y la desconfianza que inicialmente despertó este procedimiento, a lo largo de los años se ha ido abriendo paso como una opción reconstructiva efectiva que se ha posicionado en la cúspide de la escalera de técnicas reconstructivas ${ }^{18}$. En general, los logros a corto y medio plazo han sido favorables, con prometedores resultados funcionales y estéticos, y un evidente impacto positivo sobre la calidad de vida y los aspectos psicológicos de los receptores ${ }^{19}$. Los constantes avances en la planificación quirúrgica asistida por ordenador, la optimización en la extracción de los aloinjertos y en las técnicas microquirúrgicas, junto a la correcta y minuciosa selección de los candidatos, han permitido que progresivamente este procedimiento haya tenido una aplicación clínica más amplia ${ }^{20-24}$, evolucionando desde los primeros trasplantes parciales de algunas estructuras de la cara hasta los actuales TF más completos que incluyen varias unidades estéticas faciales. Hoy en día, se ha instalado en la comunidad científica el firme convencimiento de que, en cierta medida, las innovaciones quirúrgicas en el campo del TF han superado a los avances en los aspectos inmunológicos, médicos y éticos suscitados por este procedimiento. Sin embargo, no existe consenso sobre cuándo, cómo y por qué tomar la decisión de realizar un TF en vez de una reconstrucción autóloga, ni tampoco sobre los criterios de inclusión/exclusión de candidatos. Por tal motivo, tan compleja decisión debe valorarse en cada caso clínico concreto ${ }^{25}$.

Aun cuando el TF se ha consolidado como una nueva alternativa dentro del catálogo de procedimientos reconstructivos, todavía se debe considerar como un procedimiento experimental y extraordinario del cual queda mucho por aprender ${ }^{5}$. Pese a que el análisis comparativo de los datos disponibles sobre los casos clínicos de TF han mostrado unos resultados funcionales y estéticos globalmente alentadores, aún hay considerables aspectos de investigación y de aplicación clínica pendientes de definir para vislumbrar el alcance real y la verdadera dimensión del procedimiento ${ }^{10}$. El próximo reto será lograr nuevas estrategias de tratamientos inmunosupresores más seguras y eficaces, o potenciales avances en el campo de la inmunología que incluso puedan eludir la necesidad de seguir regímenes inmunosupresores de por vida, eliminándose así uno de los principales riesgos médicos y éticos asociados al TF.

Pedro Infante-Cossío

Servicio de Cirugía Oral y Maxilofacial, Hospital Universitario Virgen del Rocío. Sevilla. Departamento de Cirugía. Universidad de Sevilla, España. Director-Adjunto de la Revista Española de Cirugía Oral y Maxilofacial

\section{B I B L I O G R A F Í A}

1. Devauchelle B, Badet L, Lengelé B, Morelon E, Testelin S, Michallet M, et al. First human face allograft: early report. Lancet. 2006;368(9531):203-9. DOI: 10.1016/S0140-6736(06)68935-6.

2. Infante-Cossio P, Barrera-Pulido F, Gomez-Cia T, Sicilia-Castro D, Garcia-Perla-Garcia A, Gacto-Sanchez P, et al. Facial transplantation: a concise update. Med Oral Patol Oral Cir Bucal. 2013;18(2):e263-71. DOI: 10.4317/medoral.18552.

3. Kollar B, Tasigiorgos S, Dorante MI, Carty MJ, Talbot SG, Pomahac B. Innovations in reconstructive microsurgery: Reconstructive transplantation. J Surg Oncol. 2018;118(5):800-6. DOI: 10.1002/jso.25147. 
4. Gomez-Cia T, Sicilia-Castro D, Infante-Cossio P, Barrera-Pulido F, Gacto-Sanchez P, Lagares-Borrego A, et al. Second human facial allotransplantation to restore a severe defect following radical resection of bilateral massive plexiform neurofibromas. Plast Reconstr Surg. 2011;127(2):995-6. DOI: 10.1097/PRS.0b013e318200b00b.

5. Infante-Cossio P, Barrera-Pulido F, Gomez-Cia T, Sicilia-Castro D, Garcia-Perla-Garcia A, Gacto-Sanchez P, et al. Current role in facial allograft transplantation: what have we learned? Plast Aesthet Res. 2016;3:211-8. DOI: 10.20517/2347-9264.2016.11.

6. Lantieri L, Hivelin M, Audard V, Benjoar MD, Meningaud JP, Bellivier F, et al. Feasibility, reproducibility, risks and benefits of face transplantation: a prospective study of outcomes. Am J Transplant. 2011;11(2):367-78. DOI: 10.1111/j.1600-6143.2010.03406.x.

7. Fischer S, Kueckelhaus M, Pauzenberger R, Bueno EM, Pomahac B. Functional outcomes of face transplantation. Am J Transplant. 2015;15(1):220-33. DOI: 10.1111/ajt.12956.

8. BenMarzouk-Hidalgo OJ, Cordero E, Gómez-Cía T, Sánchez M, González-Padilla JD, Infante-Cossio P, et al. First face composite-tissue transplant recipient successfully treated for cytomegalovirus infection with preemptive valganciclovir treatment. Antimicrob Agents Chemother. 2011;55(12):5949-51. DOI: 10.1128/AAC.05335-11.

9. Lubek JE. Facial transplantation: what does the future hold? Oral Surg Oral Med Oral Pathol Oral Radiol. 2019;128(4):345-6. DOI: 10.1016/j. oooo.2019.06.776.

10. Rifkin WJ, David JA, Plana NM, Kantar RS, Diaz-Siso JR, Gelb BE, et al. Achievements and challenges in facial transplantation. Ann Surg. 2018;268(2):260-70. DOI: 10.1097/SLA.0000000000002723.

11. Tasigiorgos S, Kollar B, Krezdorn N, Bueno EM, Tullius SG, Pomahac B. Face transplantation-current status and future developments. Transpl Int. 2018;31(7):677-88. DOI: 10.1111/tri.13130.

12. Palmer WJ, Nelms L. Face transplantation for massive mandibular defects: considerations for allograft design and surgical planning. Plast Aesthet Res. 2020;7:2. DOI: 10.20517/2347-9264.2019.34.

13. Morelon E, Petruzzo P, Kanitakis J, Dakpé S, Thaunat O, Dubois V, et al. Face transplantation: partial graft loss of the first case 10 years later. Am J Transplant. 2017;17(7):1935-40. DOI: 10.1111/ajt.14218.

14. Pomahac B, Pribaz J, Eriksson E, Bueno EM, Diaz-Siso JR, Rybicki FJ, et al. Three patients with full facial transplantation. N Engl J Med. 2012;36(8):715- 22. DOI: 10.1056/NEJMoa1111432.

15. Siemionow M, Ozturk C. Face transplantation: outcomes, concerns, controversies, and future directions. J Craniofac Surg. 2012;23(1):2549. DOI: 10.1097/SCS.0b013e318241b920.

16. Diaz-Siso JR, Rodriguez ED. Facial transplantation: knowledge arrives, questions remain. Lancet. 2016;388(10052):1355-6. DOI: 10.1016/ S0140-6736(16)31396-4.

17. Lantieri L. Face transplant: a paradigm change in facial reconstruction. J Craniofac Surg. 2012;23(1):250-3. DOI: 10.1097/ SCS.0b013e318241c10b.

18. Siemionow M. The decade of face transplant outcomes. J Mater Sci Mater Med. 2017;28(5):64. DOI: 10.1007/s10856-017-5873-z.

19. Siemionow M. The miracle of face transplantation after 10 years. Br Med Bull. 2016;120(1):5-14. DOI: $10.1093 / \mathrm{bmb} / \mathrm{ldw0} 05$.

20. Gomez-Cia T, Infante-Cossio P, Sicilia-Castro D, Gacto-Sanchez P, Gonzalez-Padilla JD. Sequence of multiorgan procurement involving face allograft. Am J Transplant. 2011;11(10):2261. DOI: 10.1111/j.1600-6143.2011.03678.x.

21. Sicilia-Castro D, Gomez-Cia T, Infante-Cossio P, Gacto-Sanchez P, Barrera-Pulido F, Lagares-Borrego, et al. Reconstruction of a severe facial defect by allotransplantation in neurofibromatosis type 1: a case report. Transplant Proc. 2011;43(7):2831-7. DOI: 10.1016/j. transproceed.2011.06.030.

22. Fernandez-Alvarez JA, Infante-Cossio P, Barrera-Pulido F, Gacto-Sanchez P, Suarez-Mejias C, Gomez-Ciriza G, et al. Virtual reality AYRA software for preoperative planning in facial allotransplantation. J Craniofac Surg. 2014;25(5):1805-9. DOI: 10.1097/SCS.0000000000000989.

23. Infante-Cossio P, Sicilia-Castro D, Gomez-Cia T. Comment on: "Two stage face transplantation: a new concept in vascularized composite allotransplantation". Microsurgery. 2015;35(5):419-20. DOI: 10.1002/micr.22367.

24. Hurtado-Ruzza H, Sosa-Fajardo P, Martin-Biedma B, Pérez-Sayáns M, Infante-Cossio P, García-García A. Facial Donor Restoration: OneStep Technique. J Oral Maxillofac Surg. 2019;77(9):1905.e1-1905.e8. DOI: 10.1016/j.joms.2019.05.005.

25. Rifkin WJ, Bellamy JL, Kantar RS, Farber SJ, Diaz-Siso JR, Brecht LE, et al. Autologous reconstruction of a face transplant candidate. Craniomaxillofac Trauma Reconstr. 2019;12(2):150-5. DOI: 10.1055/s-0038-1675844. 